\title{
Assessing volatiles in three Chinese dwarf cherry cultivars during veraison and maturation using headspace-solid phase microextraction with GC-MS
}

\author{
Qingyu Cui ${ }^{1}$, Liqin Ye ${ }^{2}$, Chang-e Guo ${ }^{1}$ and Weidong $\mathrm{Li}^{1}{ }^{1}$ * \\ ${ }^{1}$ School of Chinese Materia Medica, Beijing University of Chinese Medicine, Beijing 102488, P. R. China \\ 2 The First Affiliated Hospital, Zhejiang University, School of Medicine, Hangzhou 310003, P. R. China
}


Table S1 Volatile compounds detected in 'Jing'ou 1', 'Jing'ou 2' and 'Jing'ou 3' fruits during veraison and maturation.

\begin{tabular}{|c|c|c|c|c|c|}
\hline Code & Compounds & $\mathrm{LRI}^{\mathrm{a}}$ & Jing'ou 1 & Jing'ou 2 & Jing'ou 3 \\
\hline & Alcohols & & & & \\
\hline $1^{*}$ & 1-Hexanol & 973 & $+\mathrm{b}$ & + & + \\
\hline $2^{*}$ & 1-Octen-3-ol & 1081 & + & + & + \\
\hline 3 & 5-Methyl-1-heptanol & 1099 & - & + & - \\
\hline $4 *$ & 2-Ethylhexanol & 1127 & + & + & + \\
\hline 5 & Caprylic alcohol & 1179 & + & - & + \\
\hline 6 & Cyclooctanol & 1188 & - & - & + \\
\hline 7 & 2-Octen-1-ol, $(2 E)$ - & 1189 & + & + & + \\
\hline $8^{*}$ & Benzyl alcohol & 1240 & + & + & + \\
\hline 9 & Nonyl alcohol & 1285 & + & + & + \\
\hline $10 *$ & Phenylethyl alcohol & 1324 & + & + & + \\
\hline 11 & 2-Ethyl-1-decanol & 1399 & + & + & + \\
\hline 12 & 3-Phenyl-1-propanol & 1454 & + & - & - \\
\hline 13 & Phenylacetylcarbinol, (-)- & 1480 & + & + & + \\
\hline 14 & Dihydro- $\beta$-ionol & 1590 & - & + & - \\
\hline 15 & 2,4a,5,8a-tetramethyl-1,2,3,4,7,8-hexahydronaphthalen-1-ol & 1636 & + & - & - \\
\hline \multirow[t]{2}{*}{16} & Phytol & 1917 & + & - & - \\
\hline & Carbonyls & & & & \\
\hline 17 & Senecialdehyde & 947 & + & - & + \\
\hline
\end{tabular}




\begin{tabular}{|c|c|c|c|c|c|}
\hline 18 & (Z)-2-Heptenal & 1100 & + & + & + \\
\hline 19 & 2-Heptenal, $(2 E)-$ & 1101 & + & + & + \\
\hline 20 & 1-Octen-3-one & 1110 & - & + & + \\
\hline $21 *$ & Benzaldehyde & 1152 & + & + & + \\
\hline 22 & 2,4-Heptadienal, $(2 E, 4 E)-$ & 1183 & + & + & + \\
\hline 23 & Hept-4-enal & 1184 & + & - & + \\
\hline 24 & 2-Octenal, $(2 E)-$ & 1199 & + & + & + \\
\hline $25^{*}$ & Nonanal & 1223 & + & + & + \\
\hline 26 & Acetophenone & 1281 & + & + & + \\
\hline 27 & $p$-Menth-1-en-9-al & 1384 & + & + & + \\
\hline 28 & 1-Phenyl-1,2-propanedione & 1388 & + & + & + \\
\hline 29 & 2-Decenal, $(2 E)$ - & 1399 & - & + & + \\
\hline 30 & 2-Dodecenal & 1400 & + & + & + \\
\hline 31 & 2-Undecenal & 1502 & + & + & + \\
\hline 32 & Dihydro- $\beta$-ionone & 1600 & + & + & + \\
\hline 33 & 2,4-Octadienal, $(2 E, 4 E)-$ & 1634 & - & - & + \\
\hline 34 & 4-(2,6,6-Trimethylcyclohexa-1,3-dienyl)but-3-en-2-one & 1692 & + & - & - \\
\hline \multirow[t]{2}{*}{35} & 4,8-Dimethylnon-7-en-2-one & 1734 & - & + & - \\
\hline & Esters & & & & \\
\hline 36 & Isoamyl acetate & 975 & + & + & + \\
\hline 37 & Isoprenyl acetate & 1008 & + & - & - \\
\hline 38 & Amyl acetate & 1023 & - & - & + \\
\hline
\end{tabular}




\begin{tabular}{|c|c|c|c|c|c|}
\hline 39 & Heptyl dichloroacetate & 1080 & - & - & + \\
\hline 40 & Ethyl caproate & 1108 & + & + & - \\
\hline $41^{*}$ & Hexyl acetate & 1122 & + & + & + \\
\hline 42 & Methyl benzoate & 1287 & + & + & + \\
\hline 43 & Butyl hexanoate & 1298 & + & + & + \\
\hline 44 & Ethyl octanoate & 1306 & + & + & + \\
\hline 45 & Hexyl pivalate & 1334 & + & - & - \\
\hline 46 & Hexyl 2-methylbutanoate & 1335 & + & + & + \\
\hline 47 & 3-Hexenyl isovalerate, $(3 Z)-$ & 1345 & + & + & + \\
\hline 48 & Isoamyl hexanoate & 1354 & + & + & + \\
\hline $49 *$ & Ethyl benzoate & 1362 & + & + & + \\
\hline $50 *$ & Benzyl acetate & 1377 & + & + & + \\
\hline $51^{*}$ & Prenyl acetate & 1382 & + & + & - \\
\hline 52 & 1,5-Di(methoxycarbonyloxy)pentane & 1383 & + & + & - \\
\hline 53 & Methyl salicylate & 1385 & - & - & + \\
\hline 54 & Decyl acetate & 1419 & - & - & + \\
\hline 55 & Nonyl acetate & 1420 & + & + & - \\
\hline 56 & 6-Butyloxan-2-one & 1425 & + & - & - \\
\hline 57 & {$[(E)$-But-2-enyl] hexanoate } & 1426 & + & + & + \\
\hline 58 & Prenyl caproate & 1433 & + & + & + \\
\hline 59 & Benzyl propionate & 1471 & - & - & + \\
\hline $60 *$ & Phenethyl acetate & 1473 & + & + & + \\
\hline
\end{tabular}




\begin{tabular}{|c|c|c|c|c|c|}
\hline 61 & Citronellyl acetate & 1477 & - & + & + \\
\hline 62 & Hexyl hexanoate & 1493 & + & + & + \\
\hline 63 & Butyl octanoate & 1494 & + & + & + \\
\hline 64 & Ethyl decanoate & 1501 & + & + & + \\
\hline 65 & Neryl acetate & 1511 & + & + & + \\
\hline 66 & Geranyl acetate & 1541 & - & - & + \\
\hline 67 & 2-Methylbutyl octanoate & 1548 & + & + & - \\
\hline 68 & Butyl benzoate & 1569 & + & + & + \\
\hline 69 & sec-Butyl benzoate & 1571 & - & + & - \\
\hline 70 & 3-Methylbut-2-enyl octanoate & 1629 & + & + & + \\
\hline 71 & Geranyl formate & 1630 & - & - & + \\
\hline 72 & Hexyl octanoate & 1689 & + & - & + \\
\hline 73 & Ethyl laurate & 1699 & + & + & + \\
\hline 74 & Geranyl butyrate & 1713 & + & - & + \\
\hline 75 & Ethyl cinnamate & 1715 & + & + & + \\
\hline 76 & Hexyl benzoate & 1783 & - & - & + \\
\hline 77 & Ethyl oleate & 1877 & - & + & + \\
\hline 78 & Ethyl palmitate & 1883 & + & + & + \\
\hline 79 & Ethyl hexadec-9-enoate & 1890 & + & + & + \\
\hline 80 & Ethyl tetradec-9-enoate & 1892 & + & + & + \\
\hline 81 & Ethyl myristate & 1899 & + & + & - \\
\hline 82 & Ethyl behenate & 1900 & - & - & + \\
\hline
\end{tabular}


83 Linalyl isovalerate

$1910+$

\section{Hydrocarbons}

$84 \quad$ Styrene

85 1,1,3-Trimethylcyclopentane

86 1-Ethyl-3-methylcyclopentane

87 2,3-Nonadiene

88 Isobutylcyclopentane

89 1,3-Cyclooctadiene

90 (1S,2S)-1,2-Dimethylcyclopentane

$91 \quad p$ - $\alpha$-Dimethyl styrene

92 Cycloheptane

93 5-Ethyl-m-xylene

$94 \quad o$-Cymene

95 Pentylcyclopropane

96 2,6,11-Trimethyldodecane

97 Cyclooctene, $(Z)-$

$98 \quad$ Naphthalene

99 1-Methylcyclohexa-1,4-diene

100 1-Butan-2-yl-1-(2-methylbutyl)cyclopropane

101 Undecane

$102 \quad 1-N o n e n e$

103
1020

1181

1182

1185

1186

$1214+$

1224

1225

1226

1246

1286

1299

1310

1329

1383

1384

1399

1400

1421

1424 


\begin{tabular}{|c|c|c|c|c|c|}
\hline 104 & Dodecane & 1464 & + & + & + \\
\hline 105 & 2,6-Dimethylocta-2,6-diene & 1478 & + & + & - \\
\hline 106 & Cyclopropane, octyl- & 1484 & - & + & - \\
\hline 107 & 1-Methylnaphthalene & 1499 & + & + & + \\
\hline 108 & Octadecane, 3-ethyl-5-(2-ethylbutyl)- & 1515 & + & - & - \\
\hline 109 & Cyclodecane & 1519 & - & + & - \\
\hline 110 & 1,2-Dihydro-1,1,6-trimethylnaphthalene & 1521 & + & + & + \\
\hline 111 & 2-Methylnaphthalene & 1532 & + & + & + \\
\hline 112 & Cyclododecane & 1586 & + & - & - \\
\hline 113 & 2-Ethyl-p-xylene & 1598 & + & + & + \\
\hline 114 & Hexadecane & 1599 & + & + & + \\
\hline 115 & 2-Ethylnaphthalene & 1607 & - & - & + \\
\hline 116 & 3,6-Dimethyldecane & 1631 & + & - & - \\
\hline 117 & Patchoulane & 1635 & - & + & - \\
\hline 118 & 2,3-Dimethylnaphthalene & 1650 & + & + & + \\
\hline \multirow[t]{2}{*}{119} & Heptadecane & 1700 & + & + & + \\
\hline & Lactones & & & & \\
\hline 120 & $\gamma$-Octanolactone & 1510 & + & - & - \\
\hline 121 & $\gamma$-ecalactone & 1724 & + & + & + \\
\hline \multirow[t]{2}{*}{122} & Dihydroactinidiolide & 1856 & + & + & + \\
\hline & Terpenoids & & & & \\
\hline $123 *$ & $\beta$-Pinene & 1054 & + & - & + \\
\hline
\end{tabular}




\begin{tabular}{|c|c|c|c|c|c|}
\hline 124 & Myrcene & 1055 & + & + & + \\
\hline 125 & Limonene, $(+)-$ & 1098 & + & - & + \\
\hline 126 & $\beta$-Ocimene & 1119 & + & + & + \\
\hline 127 & $\beta$-Ocimene, $(3 Z)-$ & 1120 & + & + & + \\
\hline 128 & $\alpha$-Ocimene, $(3 E)$ - & 1126 & - & - & + \\
\hline 129 & Terpinolene & 1173 & + & + & + \\
\hline 130 & (E)-linalool oxide & 1180 & - & - & + \\
\hline $131^{*}$ & Linalool & 1209 & + & + & + \\
\hline 132 & Alloocimene, $(4 E, 6 Z)-$ & 1222 & + & + & + \\
\hline 133 & Hotrienol & 1235 & + & + & + \\
\hline 134 & Nerol Oxide & 1290 & + & + & + \\
\hline 135 & Borneol & 1300 & - & + & + \\
\hline 136 & 4-Carvomenthenol & 1307 & + & + & + \\
\hline $137^{*}$ & Terpineol & 1338 & + & + & + \\
\hline 138 & Citronellol & 1359 & + & + & + \\
\hline 139 & Nerol & 1368 & + & - & - \\
\hline 140 & 2-Butynol & 1369 & - & - & + \\
\hline 141 & $\beta$-Cyclocitral & 1391 & + & + & + \\
\hline $142 *$ & Geraniol & 1401 & + & + & + \\
\hline 143 & Neral & 1409 & - & + & - \\
\hline 144 & Geranial & 1448 & + & + & + \\
\hline 145 & Carvacrol & 1489 & - & - & + \\
\hline
\end{tabular}




\begin{tabular}{|c|c|c|c|c|c|}
\hline 146 & Anethole & 1490 & + & + & + \\
\hline 147 & Estragole & 1491 & - & + & - \\
\hline 148 & Geranic Acid & 1527 & - & + & + \\
\hline 149 & Perillene & 1528 & - & + & - \\
\hline 150 & Eugenol & 1578 & + & + & + \\
\hline 151 & Geranylgeraniol & 1589 & - & + & - \\
\hline 152 & Dihydropseudoionone & 1615 & + & + & + \\
\hline & Others & & & & \\
\hline 153 & 2-Methylbutanoic acid & 953 & + & + & + \\
\hline 154 & Hexanoic Acid & 1106 & - & + & + \\
\hline 155 & Heptanoic Acid & 1109 & + & + & + \\
\hline 156 & Octanoic Acid & 1303 & + & - & + \\
\hline $157^{*}$ & Benzoic Acid & 1367 & + & + & + \\
\hline 158 & Phenol, 2-methoxy-3-(2-propenyl) & 1579 & + & + & + \\
\hline 159 & 1-But-3-en-2-yl-4-methoxybenzene & 1631 & - & - & + \\
\hline 160 & 4-Butyl-indan-5-ol & 1632 & + & + & + \\
\hline 161 & Butylated Hydroxytoluene & 1675 & + & + & - \\
\hline 162 & 2,4-DI-Tert-butylphenol & 1685 & + & + & + \\
\hline 163 & 5,5,8a-Trimethyl-3,6,7,8-tetrahydro-2H-chromene & 1767 & + & + & + \\
\hline 164 & Olivetol & 1768 & + & + & + \\
\hline
\end{tabular}

a linear retention index;

b ' + ' indicates that the volatile was detected and '-' indicated that the volatile was not detected;

* mass spectrum and retention time consistent with those of the standard compounds available in the laboratory. 